\title{
Optimal Time Period of Wearing Protective Collar after Anterior Cervical Discectomy and Fusion
}

\author{
Qian $Y^{1,2 \#, ~ Y u ~} Z^{1,2 \#, ~ L i u ~} Z^{1,2}$, Duan $W^{1,2}$, Zhao $Z^{1,2}$, \\ Zheng $H^{1,2}$ and J ian $F^{1,2 *}$ \\ ${ }^{1}$ Department of Neurosurgery, Xuanwu Hospital, Capital \\ Medical University, Beijing, China \\ ${ }^{2}$ Spine Center, China International Neuroscience Institute \\ (China-INI), China \\ ${ }^{\#}$ Contributed Equally to this Work \\ *Corresponding author: FengzengJ ian, Department \\ of Neurosurgery, Xuanwu Hospital, Capital Medical \\ University, No. 45, Changchun Street, Xicheng District, \\ Beijing, 100053, China
}

Received: May 04, 2021; Accepted: May 27, 2021;

Published: June 03, 2021

\begin{abstract}
Objective: There is still no consensus on the time period of wearing protective collar after Anterior Cervical Discectomy and Fusion (ACDF). We aim to investigate the optimal time period of wearing collar after ACDF.

Methods: We retrospectively reviewed patients with cervical spondylosis who underwent one or two segment ACDF during January 2016 and December 2017, and included 97 patients who met inclusion and exclusion criteria. Patients were divided into three groups, 1-4 week group, 5-8 week group and 9-12 week group, according to the actual time period of wearing collar after ACDF. We analyzed Japanese Orthopedic Association (JOA) score, Axial Symptom (AS) score and Neck Disability Index (NDI) before surgery and at post-operative 3 months to investigate the optimal time period of wearing collar after ACDF procedure.
\end{abstract}

Results: 1) JOA score: All three groups have a better post-operative JOA score compared with that before surgery (paired t test, $p<0.05$ ). There is no significant difference among the three groups with respect to post-operative JOA (ANOVA, p>0.05). 2) AS score: The post-operative AS scores of 1-4 week group and $5-8$ week group were significantly better than that before surgery (paired $t$ test, $p>0.05$ ). While the post-operative AS score of 9-12 week group was significantly worse than preoperative AS score (paired t test, $p<0.05)$. 3) NDI: All three groups have a better post-operative NDI compared with that before surgery (McNemar test, $p<0.05$ ). Of note, in $5-8$ weeks group, the percentage of no deficit increased by $45 \%$, and the percentage of mild deficit decreased by $45 \%$ accordingly. That percentage is $26 \%$ and $31 \%$ in $1-4$ week group and $9-12$ week group, respectively. There was significant difference among these three groups (Fisher's exact probability test, $\mathrm{p}<0.05$ ).

Conclusion: For cervical spondylosis patients who underwent 1 or 2 segment $A C D F$, the optimal time period of wearing protective is 5-8 weeks. This time period results in comparable neurological outcome, least risk of axial symptoms, and highest chance of no deficit with neck function.

Keywords: Anterior Cervical Discectomy and Fusion; Time Period; Protective Collar; Axial Symptom; Retrospective Study

\section{Introduction}

Cervical spondylosis is a common degenerative disease of the cervical spine [1]. Degeneration of the cervical intervertebral disc and its secondary pathological changes cause stimulation or compression on adjacent nerve roots, spinal cord, vertebral artery, or cervical sympathetic nerves, resulting in various symptoms such as neck pain, ataxia, numbness and weakness of limbs. With the changes in people's life and work style, especially popularization of computer and smart phone, more and more people experience long-term subhealthy cervical spine, which eventually leads to cervical spondylosis. Min et al. reported nearly 150 million people in China suffering from cervical spondylosis, including $82 \%$ of people over 60 years old, $71 \%$ of people aged $50-60$ years old and $59.1 \%$ of young adults aged $30-40$ years old [2].

For the treatment of cervical spondylosis, conservative treatments such as lifestyle modification, pain relief, and physical therapy should be tried firstly. If the symptoms are severe and refractory to conservative treatment, or myelopathy occurs, surgery is recommended. Since the Anterior Cervical Discectomy and Fusion (ACDF) was first proposed by Cloard and Robinson in the mid- $20^{\text {th }}$ century, this procedure has been widely used in clinical practice and has become a common operation for the treatment of cervical degenerative diseases [3].

Wearing a protective collar is usually recommend after ACDF to maintain the stability of the spine, promote intervertebral fusion, and provide patients with a sense of security in daily activities. However, there is still no consensus on the time period of wearing collar. There are different opinions in the literature on the time period of wearing collar after ACDF. Several studies have shown that 1or 2 segment ACDF does not require postoperative external cervical support [46]. While, other literature reported that patients who underwent 1 or 2 segment ACDF needed wearing collar for 4-12 weeks during offbed ambulation [7-10]. Therefore, it is very important to study the 
impact of different time period of wearing cervical collar after ACDF on the safety, comfort and prognosis of patients. We conducted this retrospective study to explore the optimal time period of wearing protective collar for patients who receive ACDF.

\section{Methods}

The aim of the study was to investigate the optimal time period of wearing collar after ACDF.

Patients with cervical spondylosis who were admitted to our center from January 2016 through December 2017 and underwent 1 or 2 segment ACDF were retrospectively included. Inclusion criteria: (1) Patients underwent 1 or 2 level ACDF for cervical spondylosis; (2) age >18-year old, <80-year old; (3) Semi-rigid Philadelphia collar was worn after the operation. Exclusion criteria: (1) Previous cervical operations; (2) Non-degenerative diseases such as congenital malformations, trauma, fracture, cervical kyphosis, tuberculosis, tumors, etc.; (3) Poor compliance and failure to cooperate telephone follow-up.

Included patients were divided into three groups according to the time period of wearing collar, which are 1-4 week group, 5-8 week group, and 9-12 week group. Japanese Orthopedic Association (JOA), Axial Symptom (AS) and Neck Disability Index (NDI) scores were obtained pre-operatively and 3 months post-operatively.

Statistics was performed with SPSS software (Version 22, IBM, USA). Measurement data were expressed in the form of mean \pm standard deviation. Paired $t$ test was used to compare the preoperative-postoperative scores; The comparison between multiple groups was performed by one-way Analysis of Variance (ANOVA). Tukey's test was used for the post-hoc comparison; Numeration data were expressed as frequency. Chi-square test, McNemar test or Fisher exact probability test was selected as per distribution of the data. $\mathrm{P}$ $<0.05$ indicates statistically significant difference.

\section{Results}

97 patients were included, including 47 females and 50 males, aged 38-76 years, with an average of $56.4 \pm 13.1$ years. Table 1 lists demographic details for the three groups. There were 39 patients in 1-4 week group, 22 patients in 5-8 week group, and 36 patients in 9-12 week group. There was no statistical difference in age, gender, BMI, drinking history, smoking history, hypertension, diabetes, coronary heart disease among the three groups of patients $(\mathrm{P}>0.05)$, indicating that the three groups of patients are comparable.

JOA score: The results of intra-group analysis showed that the JOA scores of the three groups after operation were significantly improved compared with those before operation (paired t-test, $\mathrm{p}<0.05$ ). Since there was no difference in the preoperative JOA baseline of the three groups (ANOVA analysis, $\mathrm{P}>0.05$ ), postoperative JOA score of each group can be further compared. There is no statistical difference between the three groups (ANOVA analysis, $\mathrm{P}>0.05$ ), indicating that the time period of wearing collar has no significant effect on neurological rehabilitation (Figure 1, Table 2).

AS score: Intra-group analysis showed that the postoperative AS scores of the patients in the 1-4 week group and the 5-8 week group increased compared with those at baseline (paired $t$ test, $\mathrm{p}<0.05$ ). While the postoperative AS score of 9-12 week group decreased (paired t test, $\mathrm{P}<0.05$ ), suggesting that wearing collar for 9-12 weeks will increase the risk of axial symptoms (Figure 1). Since there was no difference in the baseline AS scores of the three groups (ANOVA analysis, $\mathrm{P}>0.05$ ), the postoperative AS scores of each group were further compared. The AS score of 9-12 week group was lower than that of 1-4 week group (ANOVA Analysis, Tukey's test, $\mathrm{P}<0.05$ ), while there was no statistically significant difference between the other groups (ANOVA analysis, Tukey's test, $\mathrm{P}>0.05$ ). The incidence of axial symptoms of patients in the 1-4 week group, 5-8 week group, and 9-12 week group were $10.3 \%, 13.6 \%$ and $22.2 \%$, respectively. The incidence of new axial symptoms was $19.4 \%$ (7/36) for 9-12 week group (Table 2). Therefore, the time period for wearing collar should not exceed 8 weeks.

NDI score: Intra-group analysis showed that the postoperative NDI scores of the three groups were significantly improved compared with that preoperatively $(\mathrm{McNemar}$ test, $\mathrm{P}<0.05)$. Since the baseline of the preoperative NDI of the three groups was comparable (chisquare test, $\mathrm{P}>0.05$ ), inter-group comparison of postoperative NDI scores between groups can be made. In 5-8 week group, patients with no deficit (as per NDI score) increased by $45 \%$ post-operatively, and the proportion of patients with mild deficit decreased by $45 \%$ accordingly, which is significantly better that that of 1-4 week group (26\%) and 9-12 week group (31\%) (Figure 2, Table 2).

\section{Discussion}

There is no consensus on the time period of wearing protective collar after ACDF. Short wearing time may be criticized for poor spinal stability and low fusion rate. On the contrary, patients who wear it for a long time may experience discomfort or even complications such as axial symptoms, in which cervical muscle atrophy, stiffness and contracture of the ligament and joint capsule tissue leading to neck pain, soreness, and stiffness [7]. Neck pressure may interfere

Table 1: Demographic characteristics of the three groups.

\begin{tabular}{|c|c|c|c|c|c|}
\hline Groups & $1-4$ week $(n=39)$ & $5-8$ week $(n=22)$ & $9-12$ week $(n=36)$ & $p$ & Test method \\
\hline Age (years) & $53.0 \pm 15.8$ & $60.8 \pm 9.4$ & $57.9 \pm 10.3$ & 0.062 & ANOVA \\
\hline BMI $\left(\mathrm{kg} / \mathrm{m}^{2}\right)$ & $25.4 \pm 3.4$ & $24.4 \pm 2.0$ & $24.5 \pm 3.2$ & 0.373 & ANOVA \\
\hline Gender (m:f) & $22: 17$ & $10: 12$ & 18:18 & 0.693 & Chi square \\
\hline Alcohol his. (y:n) & $5: 34$ & $2: 20$ & $3: 33$ & 0.911 & Fisher \\
\hline HTN his. (y:n) & $10: 29$ & $6: 16$ & $9: 27$ & 1 & Fisher \\
\hline DM his. (y:n) & $4: 35$ & $2: 20$ & $3: 33$ & 1 & Fisher \\
\hline CHD his. (y:n) & $3: 36$ & $1: 21$ & $4: 32$ & 0.724 & Fisher \\
\hline
\end{tabular}

m: male; f: female; y: yes; n: no; his: history; HTN: Hypertension; DM: Diabetes Mellitus; CHD: Coronary Heart Disease; Fisher: Fisher exact probability test. 


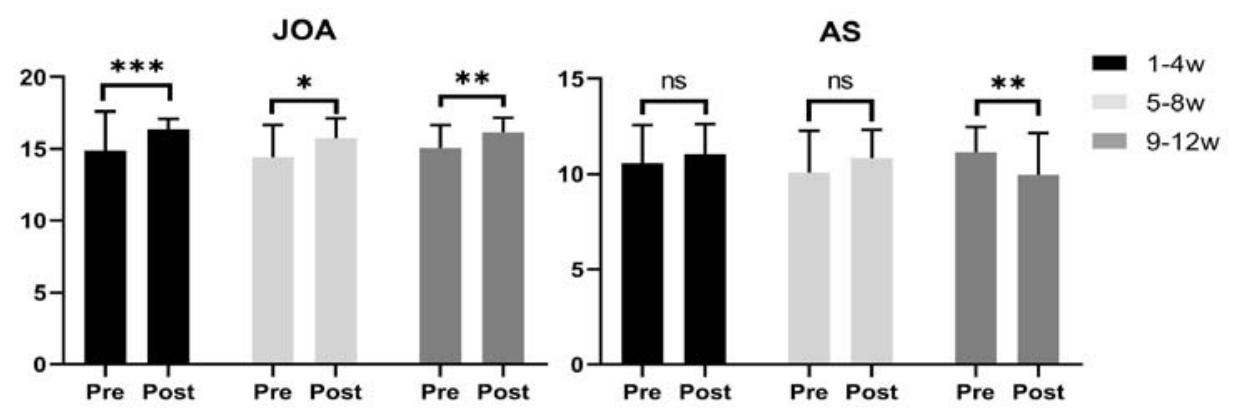

Figure 1: Pre- versus post-operative AS score and JOA score in each group. (ns: no statistically significant difference. P<0.05, statistically significant difference; ${ }^{*}<0.05$; ${ }^{*}<0.01$; ${ }^{* *}<0.001$ ); JOA: Japanese Orthopedic Association Scale; AS: Axial Symptom score; Pre-: Preoperative; Post-: Postoperative 3 months.
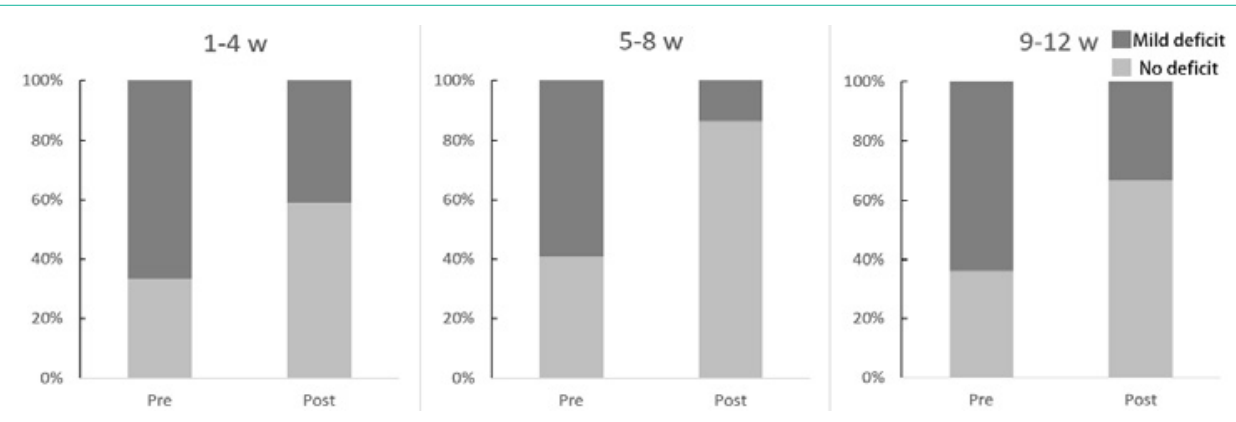

Figure 2: Pre- versus post-operative NDI score in each group. NDI: Neck Disability Index; Pre-: Preoperative; Post-: Postoperative 3 months.

Table 2: JOA, AS and NDI outcomes of the three groups.

\begin{tabular}{|c|c|c|c|}
\hline Groups & $\mathbf{1 - 4}$ week $(\mathbf{n}=\mathbf{3 9})$ & $\mathbf{5 - 8}$ week $(\mathbf{n}=\mathbf{2 2})$ & $\mathbf{9 - 1 2}$ week $(\mathbf{n = 3 6})$ \\
\hline Pre-JOA & $14.9 \pm 2.7$ & $14.4 \pm 2.3$ & $15.1 \pm 1.6$ \\
\hline Post-JOA & $16.4 \pm 0.7$ & $15.7 \pm 1.4$ & $16.1 \pm 1.0$ \\
\hline Pre-AS & $10.5 \pm 2.0$ & $10.1 \pm 2.2$ & $11.1 \pm 1.3$ \\
\hline Post-AS & $11.0 \pm 1.6$ & $10.8 \pm 1.5$ & $10.0 \pm 2.2$ \\
\hline Pre-NDI (\%)/N:M & $12.4 \pm 8.1 / 13: 26$ & $14.6 \pm 8.4 / 9: 13$ & $14.2 \pm 7.4 / 13: 23$ \\
\hline Post-NDI (\%)/N:M & $6.9 \pm 5.7 / 23: 16$ & $5.4 \pm 5.2 / 19: 3$ & $6.1 \pm 7.5 / 24: 12$ \\
\hline
\end{tabular}

Pre-: Preoperative; Post-: Postoperative 3 months; JOA: Japanese Orthopedic Association Scale; AS: Axial Symptom score; NDI: Neck Disability Index; N,M Number of patients with No deficit versus that with Mild deficit as per NDI.

with normal lymphatic and venous return, causing tissue edema after surgery and finally airway obstruction [7].

The current common practice for a surgeon is to dictate a patient to wear collar according to their personal experience, rather than a consensus or evidence-based recommendation. This study retrospectively analyzed the actual time period of wearing collar and the clinical outcomes of 97 patients who underwent one or two segment ACDF procedure and found that the optimal time period for wearing protective collar after ACDF is 5-8 weeks, which can provide the best balance of safety and comfort for the patient.

We analyzed the commonly used JOA, AS, NDI scores according different time period (1-4 week, 5-8 week, and 9-12 week) of wearing protective collar after ACDF. Comparison of JOA scores across the three groups shows that different time period has no statistically significant difference as per the rehabilitation of nerve function. Analyzing AS scores across the three group shows that AS score in 9-12 week group decreased significantly compared with 1-4 week or
5-8 week group. The incidence of axial symptoms was $10.3 \%, 13.6 \%$ and $22.2 \%$ in 1-4 week, 5-8 week and 9-12 week group, respectively $(\mathrm{p}<0.05$, ANOVA). Therefore, from the AS score perspective, time period of wearing collar after ACDF should not exceed 8 weeks so as not to increase the risk of axial symptoms. Finally, according to NDI scores among the three groups, the percentage of patients with no deficit increased by $26 \%, 45 \%$ and $31 \%$ in $1-4$ week, $5-8$ week and 9-12 week group, respectively ( $\mathrm{p}<0.05$, Fisher exact test). To sum up, these results show that the optimal time period of wearing protective collar after ACDF is 5-8 weeks. This time period results in comparable neurological outcome (as per JOA score), least risk of axial symptoms (as per AS score), and highest chance of no deficit with neck function (as per NDI score).

Previous literatures have different opinions on the time period of wearing protective collar for patients underwent 1 or 2 segment ACDF. A randomized control study conducted by Overley et al. showed that there is no advantage to wearing a cervical collar for 6 weeks after surgery in patients with 1 or 2 level ACDF with respect to 1-year outcome scores, 1-year fusion rates, and 6-month subsidence [5]. Karikari et al. performed a systemic review and concluded that there is no strong evidence supporting routine use of postoperative collar after 1 or 2 segments ACDF [4]. These are consistent with the conclusion in this study that the length of time period of wearing collar has no significant effect on the JOA score at 3 months after surgery. Abbott et al. conducted a randomized controlled pilot trial which showed that wearing a neck brace for 6 weeks after surgery can significantly reduce NDI and the level of neck pain [8]. There are also studies that support usage of protective collar for 4-12 weeks after 1-2 level ACDF [9-11], which is partially consistent with the optimal 5-8 week wearing time in this study. 
Several limitations exist in literature and our study. There is enormous heterogeneity among studies published, which accounts for different conclusions. Our study has a relatively small sample size, and time period partition is to some extent arbitrary. Further studies with large sample size, prospective design, and more refined time periods are warranted.

\section{Conclusions}

Our study showed that the optimal time period of wearing protective collar after 1 or 2 segments ACDF is 5-8 weeks. This time period results in comparable neurological outcome, least risk of axial symptom, and highest chance of no deficit with neck function.

\section{Highlights}

- There is still no consensus on the time period of wearing protective collar after anterior cervical discectomy and fusion (ACDF)

- The optimal time period of wearing protective collar is 5-8 weeks after ACDF.

- This time period results in comparable neurological outcome, least risk of axial symptoms, and highest chance of no deficit with neck function.

\section{Funding}

The study was Supported by Nursing Fund of Xuanwu Hospital, Capital Medical University, XWHL-2018002 (Y.Q), Science and Technology Innovation Capacity Building-Advanced Discipline Construction Project, 1192070315 (F.J), and Beijing Municipal Excellent Talents Training Fund, 2018000020124G142 (Z.L).

\section{Author's Contribution}

(F.J and Y.Q) designed the study. (Z.Z and H.Z) collected the data. (Y.Q and Z.Y) analyzed the data. (Z.Y, Z.L) prepared the manuscript. All authors approved the final version for submission.

\section{References}

1. Theodore N. Degenerative cervical spondylosis. N Engl J Med. 2020; 383 : 159-168.

2. MIN Y. Design and Use of New Fixed Cervical Pillow. China Heal Stand Manag. 2015; 24-25.

3. Woods BI, Hilibrand AS. Cervical radiculopathy: Epidemiology, etiology, diagnosis, and treatment. Journal of Spinal Disorders and Techniques. Lippincott Williams and Wilkins. 2015; 28: E251-E259.

4. Karikari I, Ghogawala Z, Ropper AE, Yavin D, Gabr M, Goodwin CR, et al. Utility of Cervical Collars Following Cervical Fusion Surgery. Does It Improve Fusion Rates or Outcomes? A Systematic Review. World Neurosurg. 2019; 124: 423-429.

5. Overley SC, Merrill RK, Baird EO, Meaike JJ, Cho SK, Hecht AC, et al. Is Cervical Bracing Necessary After One- and Two-Level Instrumented Anterior Cervical Discectomy and Fusion? A Prospective Randomized Study. Glob Spine J. 2018; 8: 40-46.

6. Bible JE, Biswas D, Whang PG, Simpson AK, Rechtine GR, Grauer JN. Postoperative bracing after spine surgery for degenerative conditions: a questionnaire study. Spine J. 2009; 9: 309-316.

7. Abram S, Bulstrode C. Routine spinal immobilization in trauma patients: What are the advantages and disadvantages? Surgeon. 2010; 8: 218-222.

8. Abbott A, Halvorsen M, Dedering $\AA$. Is there a need for cervical collar usage post anterior cervical decompression and fusion using interbody cages? A randomized controlled pilot trial. Physiother Theory Pract. 2013; 29: 290-300.

9. Caplan I, Sinha S, Schuster J, Piazza M, Glauser G, Osiemo B, et al. The utility of cervical spine bracing as a postoperative adjunct to single-level anterior cervical spine surgery. Asian J Neurosurg. 2019; 14: 461.

10. Wright IP, Eisenstein SM. Anterior Cervical Discectomy and Fusion without Instrumentation. Spine (Phila Pa 1976). 2007; 32: 772-774.

11. Wei Z, Wang Y, Wei Y, Bai L, Li Y, Li Y. Observation of Zero-P Zero Profile Anterior Cervical Interbody Fusion Fixation System for the Treatment of Cervical Spondylosis. China Heal Stand Manag. 2016; 7: 67-69. 